\title{
LA EVOLUCIÓN GLACIAR DE SIERRA NEVADA YLA FORMACIÓN DE GLACIARES ROCOSOS ${ }^{1}$
}

\author{
Antonio Gómez Ortiz ${ }^{(1)}$, David Palacios ${ }^{(2)}$, Bogdan Palade( ${ }^{(3)}$, Lorenzo Vázquez Selem ${ }^{(4)}$, \\ Ferran Salvador Franch ${ }^{(1)}$, Luis Miguel Tanarro García ${ }^{(2)}$ y Marc Oliva Franganillo( ${ }^{(5)}$ \\ (1) Departamento de Geografía Física y Análisis Geográfico Regional. Universidad de Barcelona \\ gomez@ub.edu \\ (2) Departamento de Análisis Geográfico Regional y Geografía Física. Universidad Complutense de Madrid \\ davidp@ghis.ucm.es \\ (3) Centro de Satélites de la Unión Europea (EUSC). Torrejón de Ardoz (Madrid) \\ (4) Instituto de Geografía. Universidad Nacional Autónoma de México \\ (5) Departamento de Geografía. Universidad de Lisboa
}

\section{RESUMEN}

El trabajo estudia cuatro valles glaciados de Sierra Nevada del área Veleta-Mulhacén $\left(37^{\circ} 3^{\prime} \mathrm{N}, 3^{\circ} 21^{\prime} \mathrm{W},>3.300 \mathrm{~m}\right)$. El análisis del isótopo cosmogénico ${ }^{36} \mathrm{Cl}$ de 19 muestras indica que el último máximo avance glaciar local fue dentro del MIS 2. El retroceso generalizado de los glaciares ocurrió a partir de 15/14 ka. Justo después se formó un extenso sistema de glaciares rocosos hasta hace $7 \mathrm{ka}$. El origen de estas formas está en relación con el proceso de deglaciación y la gran actividad geomorfológica de las paredes de los circos donde se albergaron y no tanto por un clima periglaciar extremo.

Palabras clave: Último Máximo Avance Glaciar, Deglaciación, Glaciares rocosos, dataciones cosmogénicas, Sierra Nevada, Pleistoceno tardío, Holoceno.

\section{ABSTRACT}

This paper studys four glaciated valleys around the Pico del Veleta-Pico Mulhacén $\left(37^{\circ} 3^{\prime} \mathrm{N}, 3^{\circ} 21^{\prime} \mathrm{W},>3,300 \mathrm{~m}\right)$. The analyses of 19 samples cosmogenic isotope ${ }^{36} \mathrm{Cl}$ shows that the last local glacial maximum advance was within the MIS 2. The general retreat of the glaciers was in 15/14 ka. Just after this, an extensive system of rock glaciers were formed,

Fecha de recepción: mayo 2011.

Fecha de aceptación: diciembre 2012. 
lasting until $7 \mathrm{ka}$. The origin of these rock ${ }^{1}$ glaciers is related to the deglaciation process and the considerable geomorphologic activity of the walls rather than caused by extreme periglacial climate.

Key words: Last Glacial Maximum, deglaciation, rock glacier, cosmogenic dating, Sierra Nevada, late Pleistocene, Holocene.

\section{INTRODUCCIÓN}

En la última década se está avanzando rápidamente en el conocimiento de la máxima extensión que tuvieron los glaciares en la última glaciación en las montañas del sur de Europa y en las distintas fases de su deglaciación (Hughes et al., 2006a, 2006b, 2007; Hughes y Woodward, 2008; García-Ruiz et al., 2010).

A pesar de los grandes progresos en el conocimiento de la cronología glaciar del sur de Europa quedan aún montañas muy significativas por estudiar. Este es el caso de Sierra Nevada, distante tan sólo a $170 \mathrm{~km}$ de África, cuyos últimos glaciares desaparecieron en el siglo pasado. El objetivo de este trabajo es estudiar, a través del análisis geomorfológico, sus formas glaciares y periglaciares más significativas y, al tiempo, obtener dataciones absolutas fiables del proceso de deglaciación mediante el estudio del isótopo cosmogénico ${ }^{36} \mathrm{Cl}$ y relacionarlo con la extinción glaciar histórica de la Pequeña Edad de Hielo, atestiguada por fuentes documentales históricas.

Sierra Nevada forma parte de las Cordilleras Béticas. Está instalada en el extremo suroriental de la península Ibérica, cercana al mar Mediterráneo y con dirección SW-NE. Esta Sierra, con una longitud de unos $93 \mathrm{~km}$, incluye las cumbres de mayor altitud de la península

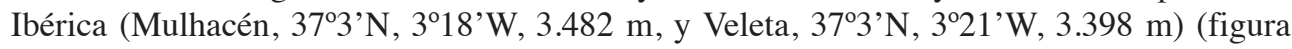
1). Está compuesta por rocas metamórficas paleozoicas (micasquistos, pizarras, cuarcitas y gneises). La temperatura media anual del aire, a $2.500 \mathrm{~m}$, es de $4,4^{\circ} \mathrm{C}$ y la precipitación de $750 \mathrm{~mm}$, de la que el $40 \%$ es en forma de nieve (Montávez et al., 1996). Esta temperatura, a partir de 3300 metros, es negativa $\left(-0,4^{\circ} \mathrm{C}\right.$, a $\left.3398 \mathrm{~m}\right)$. La oscilación térmica del suelo a los -5 cm de profundidad es muy amplia (a $3.398 \mathrm{~m}$, entre $56,4^{\circ} \mathrm{C}$ y $48,2^{\circ} \mathrm{C}$ ), pero a partir de los -20 metros queda estabilizada en torno los $2^{\circ} \mathrm{C}$ durante todo el año ( Gómez Ortiz et al., 2002; Salvador-Franch et al., 2010, 2011).

En Sierra Nevada se desarrollaron los sistemas glaciares más meridionales de Europa. Su morfología se reparte en una superficie aproximada de $326 \mathrm{~km}^{2}$, en cabeceras de valles, por encima de los $2.500 \mathrm{~m}$ de altitud, entre el puerto de Trevélez $(2.812 \mathrm{~m})$ y el cerro del Caballo $(3.013 \mathrm{~m})$. En cualquier caso se trató de modestos glaciares de valle y de circo muy supeditados a la disposición de la red hidrográfica preexistente ( Gómez Ortiz et al., 2002). Los mayores glaciares, con una orientación septentrional, tuvieron una longitud en tono a los $10-12 \mathrm{~km}$.

1 Este trabajo se ha realizado dentro del Proyecto de Investigación 018/2007 del Organismo Autónomo de Parques Nacionales del Ministerio de Medio Ambiente, cuyo Investigador Principal es el Dr. Antonio Gómez Ortiz. 
Figura 1

LOCALIZACIÓN DEL ÁREA DE ESTUDIO

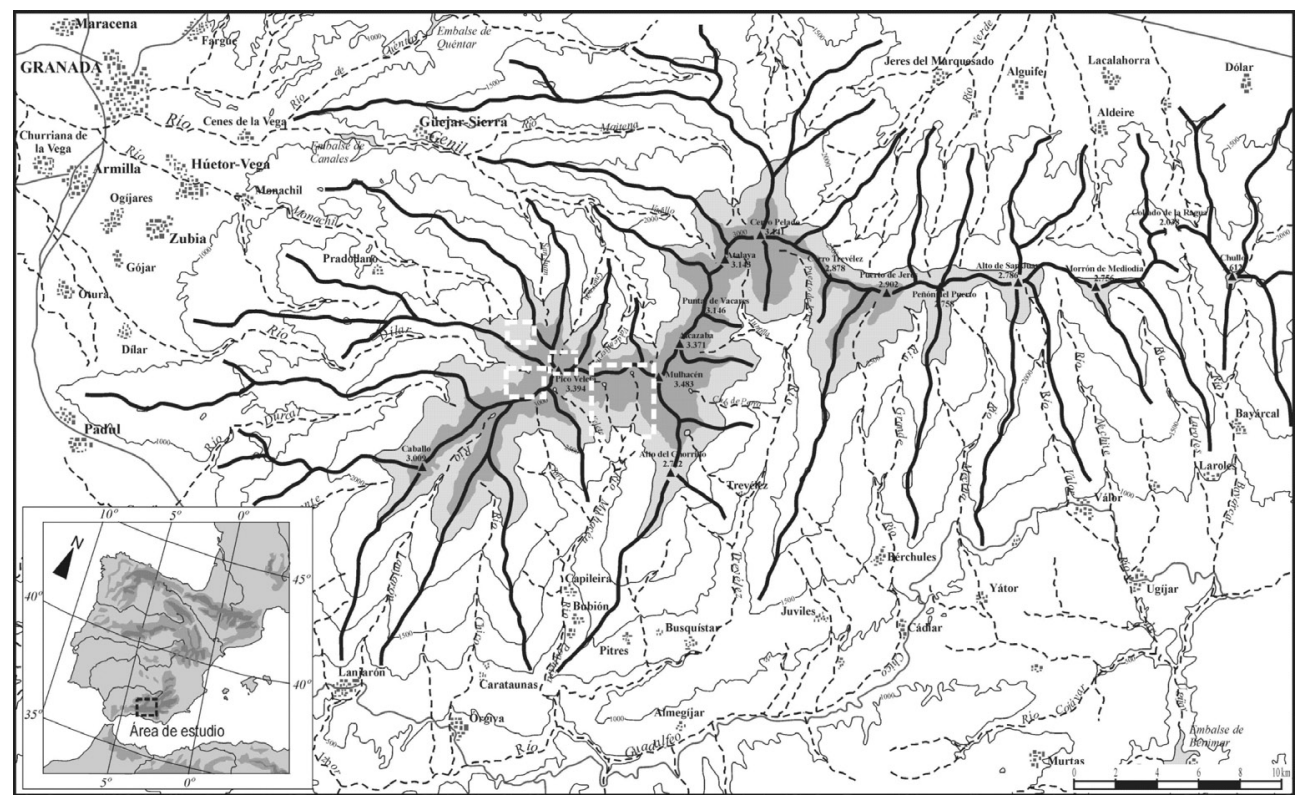

El interés por el conocimiento del glaciarismo cuaternario de Sierra Nevada (figura 2) comenzó a mediados del siglo XIX (Schimper, 1849; MacPherson, 1875), aunque la detallada descripción de las formas glaciares se realizó a lo largo del siglo XX, delimitando, describiendo y proponiendo cronología relativa de los grandes complejos morrénicos en los diferentes valles de la Sierra (Quelle, 1908; Obermaier, 1916; García-Sainz, 1947; Casas, 1943; Paschinger, 1957; Hempel, 1960; Messerli, 1965; Lhenaff, 1977; Sánchez-Gómez, 1990). Gómez Ortiz et al. (2002) ofrecieron una cartografía de la geomorfología glaciar y periglaciar de detalle de todo el conjunto de la Sierra. La mayoría de estos autores defienden la existencia de restos morrénicos anteriores a la última glaciación, muchos de ellos muy deteriorados por la erosión (Obermaier, 1916; García-Sainz, 1943; Paschinger, 1957; Hempel, 1960; Messerli, 1965; Lhenaff, 1977; Sánchez-Gómez, 1990; Gómez Ortiz et al., 2002). Hempel (1960) y Messerli (1965) proponen la altitud de las nieves perpetuas durante los máximos avances de los glaciares anteriores a la última glaciación entre 2.000 y $2.100 \mathrm{~m}$. Diversos autores proponen un nivel de nieves perpetuas durante la última glaciación entre los 2.300 y $2.500 \mathrm{~m}$, dependiendo de las orientaciones, mientras que los mismos autores proponen una altitud de entre 2.800 y $2.900 \mathrm{~m}$ del nivel de las nieves perpetuas durante re-avances tardíos, al final de la glaciación (Obermaier, 1916; Casas, 1943; Paschinger, 1957; Hempel, 1960; Messerli, 1965).

La atención prestada a los glaciares rocosos de Sierra Nevada es mucho más limitada, resumida, en principio, a escuetas referencias a su existencia (Messerli, 1965; Lhenaff, 1977). Posteriormente, Soria y Soria (1986) los clasifican y proponen una cronología Tardiglaciar 
Figura 2

POSIBLE EXTENSIÓN DE LOS GLACIARES EN SIERRA NEVADA DURANTE EL «ÚLTIMO MÁXIMO AVANCE GLACIAR NEVADENSE (UMGN)» E INDICIOS DE AVANCES MAYORES PREVIOS

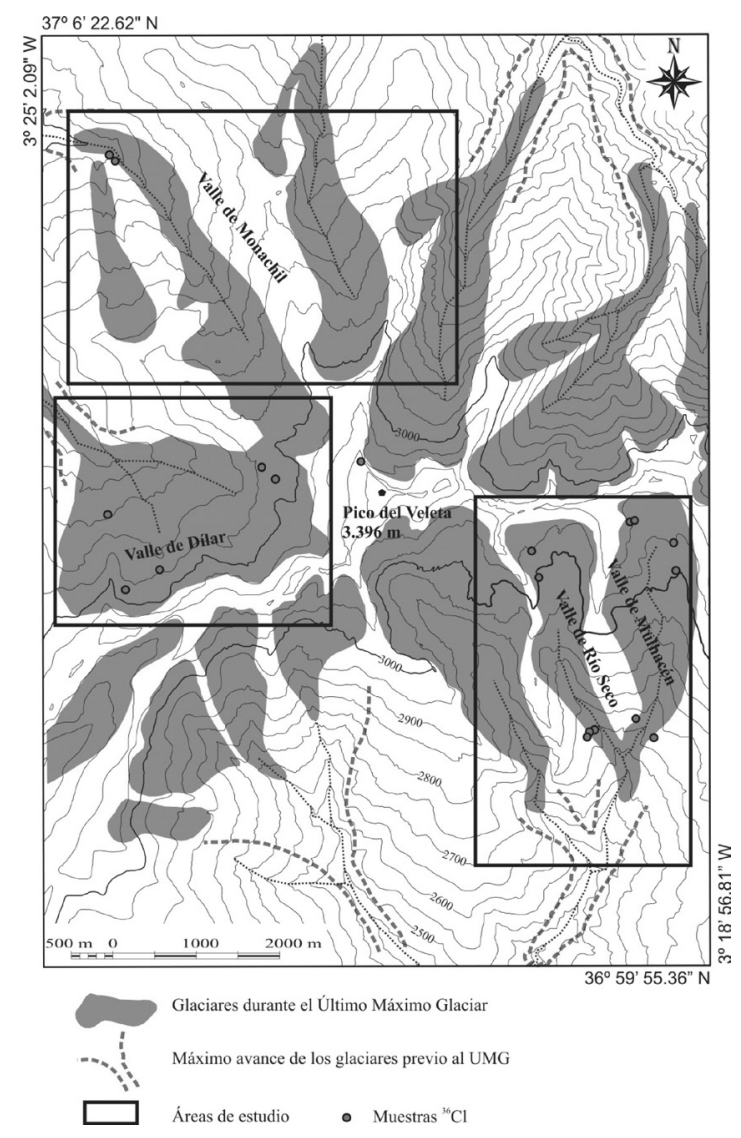

para ellos y Gómez Ortiz et al. (2002), los interpretan dentro del contexto de la morfología periglaciar de la Sierra resaltándolos en su cartografía geomorfológica.

En cuanto al glaciarismo histórico de la Pequeña Edad del Hielo hay constancia de su existencia. Consistió en pequeños focos instalados en la base de los circos de la Sierra protegidos por grandes paredes ( Gómez Ortiz et al., 2009). El más importante fue el glaciar del Corral del Veleta, situado en el circo del Guarnón, al pie de la pared norte del pico del Veleta. Ya a mediados del siglo XVIII comienza a darse información de sus masas heladas (Ponz, 1797) y desde entonces se describe con detalle por diversos autores, incluso dando medidas de su magnitud (Boissier, 1839; Hellmann, 1881; Willkomm, 1882; Rute, 1889; Quelle, 1908). Según estas descripciones el glaciar tendió a disminuir su extensión a mediados del siglo XIX ( Gómez Ortiz et al., 2008). Su retroceso se acelera durante el siglo XX (Obermaier, 1916), hasta considerarlo, a finales de la primera mitad de ese siglo, como una pequeña masa de hielo inmovilizada y decrépita (Sermet, 1942; García-Sainz, 1947; Messerli, 1967). 
Recientes estudios han detectado en el Corral del Veleta la existencia de hielo relicto bajo los mantos de derrubios situados en la base y talud de la pared norte del Pico del Veleta, en el lugar que ocupara el glaciar ( Gómez Ortiz et al., 2001; Tanarro et al., 2001). Parte de los paquetes de derrubios de este talud han adoptado la forma de glaciar rocoso en la medida que la masa de hielo ha ido disminuyendo ( Gómez Ortiz et al., 2008). En la actualidad, este glaciar rocoso continúa desplazándose, aunque predominando los movimientos de colapso, frente a los de avance (Sanjosé et al., 2007). Estudios derivados del análisis de la dinámica del talud del Corral del Veleta y de su origen a partir del retroceso del hielo, proponen un modelo de comportamiento, donde la gran actividad de la pared de pico del Veleta, afectada por constantes desprendimientos, y la disminución del aporte nival serían la causa de la transformación de un glaciar en un glaciar rocoso (Gómez Ortiz et al., 2008).

\section{METODOLOGÍA}

En primer lugar, se realizó un detallado estudio geomorfológico de las formas glaciares y de los glaciares rocosos en tres de los principales valles glaciares situados en el sector de cumbres más elevado de Sierra Nevada, con orientaciones contrastadas. La cabecera del valle de Poqueira, en las laderas meridionales del pico del Mulhacén, y los valles de Dílar y Monachil, en las laderas occidentales del Pico del Veleta. A partir de este estudio, se delimitaron y cartografiaron los modelados glaciares poco o nada alterados por la erosión mecánica. La obtención de un mapa de formas glaciares estables permitió seleccionar y muestrear registros de superficies significativas y calibrar su datación por ${ }^{36} \mathrm{Cl}$.

Figura 3

TOMA DE MUESTRAS PARA ${ }^{36}$ CL EN LAS MORRENAS DEL MÁXIMO AVANCE LOCAL EN EL VALLE DEL MULHACÉN (MUESTRA MULH-M-2)

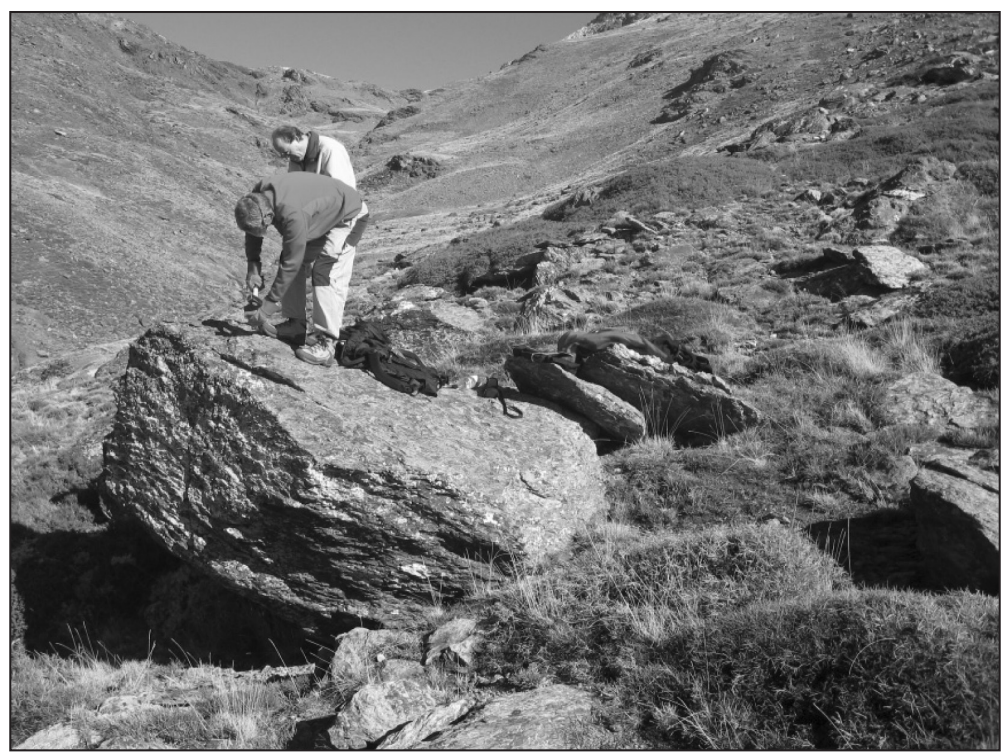


Las muestras se obtuvieron de bloques de más de $1 \mathrm{~m}$ de eje mayor (figura 3). Los bloques seleccionados fueron los más estables, situados en laderas de suave pendiente. También se tomaron muestras de roca madre en umbrales afectados por pulimiento glaciar. El procedimiento del laboratorio fue el señalado por Zreda et al. (1999) y Phillips (2003). Las edades de exposición fueron calculadas usando el programa CHLOE (Phillips y Plummer, 1996, version 3-2003), con los parámetros de producción de neutrones para ${ }^{36} \mathrm{Cl}$ propuesto por Phillips et al. (2001); la producción de muones según Stone et al. (1998); y la escala de altitudes la indicada por Lal (1991). Las edades de exposición fueron calculadas con el supuesto de 0 (no erosión), 3 y 5 mm/1.000 años de erosión.

\section{CARACTERÍSTICAS GEOMORFOLÓGICAS}

\section{Valle de Poqueira}

El río Poqueira drena la vertiente sur de Sierra Nevada, entre el Pico del Veleta y el Pico del Mulhacén (figuras 4 y 5). Su cabecera está organizada a partir de tres afluentes, de oeste a este: los ríos Veleta, Seco y Mulhacén. Los valles están modelados por formas glaciares. Su perfil es escalonado y con frecuencia, en cabecera, aparecen cubetas de sobre-excavación que albergan pequeñas lagunas limitadas por umbrales rocosos, con claro pulimiento glaciar. Quedan evidencias de formaciones morrénicas que confirman que las lenguas glaciares que descendían por cada uno de los tres barrancos llegaron a confluir en una sóla lengua, tras haber cubierto una longitud superior a los $9 \mathrm{~km}$. En efecto, fue en el lugar llamado la Hoya del Capitán, donde aún perduran restos de morrenas muy alteradas por la erosión, no adecuadas para la obtención de dataciones cosmogénicas. Aguas arriba, en cada valle, existen morrenas laterales de un gran avance posterior, muy bien conservadas, similares a las que en otras montañas europeas se han considerado pertenecientes al Último Máximo Glaciar y que, en Sierra Nevada, al no conocerse su fecha, podríamos definir como Último Máximo Glaciar Local (UMGL).

En los casos de los barrancos de Río Seco y Mulhacén, la disposición de estas morrenas laterales muestran que en su avance llegaron a fusionarse cuando cada glaciar había recorrido en torno a los $5 \mathrm{~km}$. Más arriba, en los circos, aparecen formaciones morrénicas laterales posteriores a lo que hemos denominado UMGL, indicando diversas fases de avance o estabilización durante el retroceso glaciar. En el caso del barranco del Mulhacen, justo debajo de su pared de cabecera, aparece una formación morrénica que cierra el circo y retiene la laguna de la Caldera, formada por grandes bloques, con escaso material fino. Esta morrena se apoya sobre umbrales rocosos con pulimiento glaciar. La cabecera de Río Seco es muy similar, con lagunas y umbrales pulidos, pero sin que exista una morrena tardía semejante.

En los tres barrancos citados aparecen, siempre en su ladera oriental, glaciares rocosos en el interior de pequeños circos laterales, con una longitud media en torno a los $500 \mathrm{~m}$. Algunos de ellos se apoyan en las formaciones morrénicas o en umbrales rocosos con pulimento glaciar. Todos estos glaciares rocosos son relictos, aunque se conservan en muy buen estado, sin haber sido afectados por la erosión posterior. 
Figura 4

COMPLEJOS MORRÉNICOS DEL UMGL EN LA CONFLUENCIA DE LOS VALLES MULHACÉN Y RÍO SECO

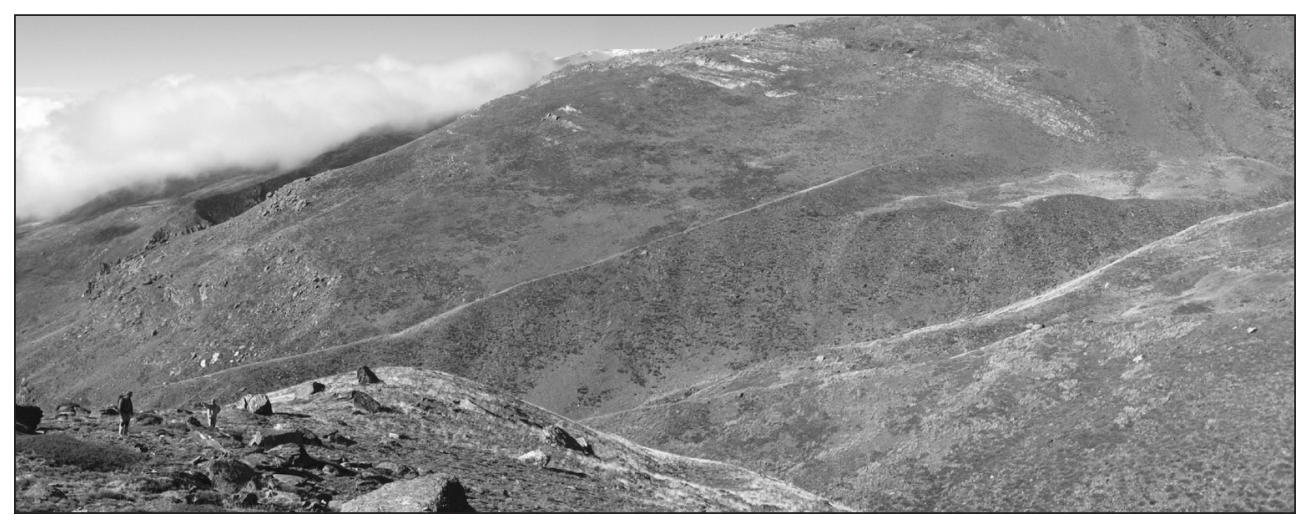

Figura 5

ESQUEMA GEOMORFOLÓGICO DE LOS VALLES RÍO SECO Y MULHACÉN, MUESTRAS Y RESULTADOS

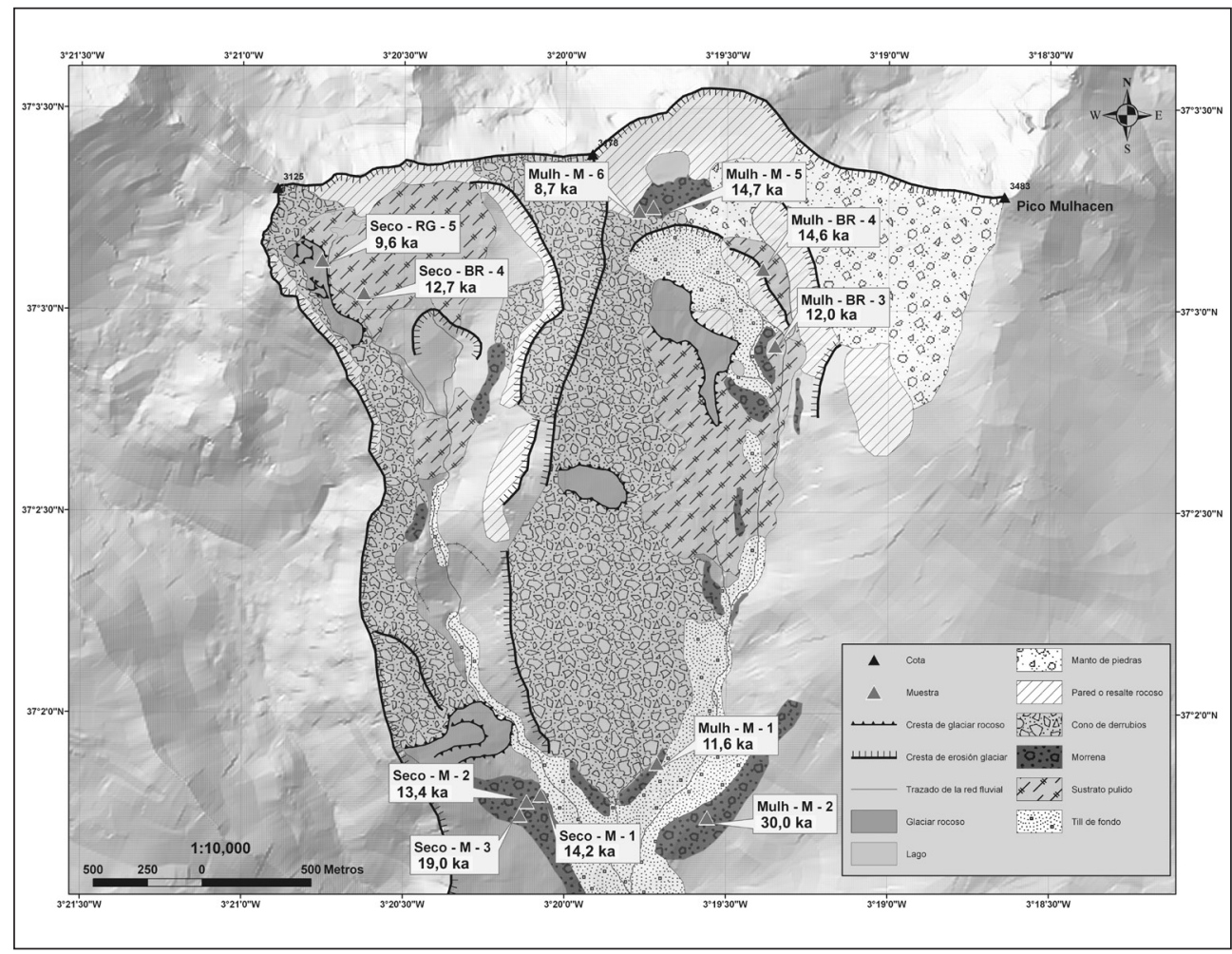


Figura 6

ESQUEMA GEOMORFOLÓGICO DE LA CABECERA DEL VALLE DE DILAR, MUESTRAS Y RESULTADOS

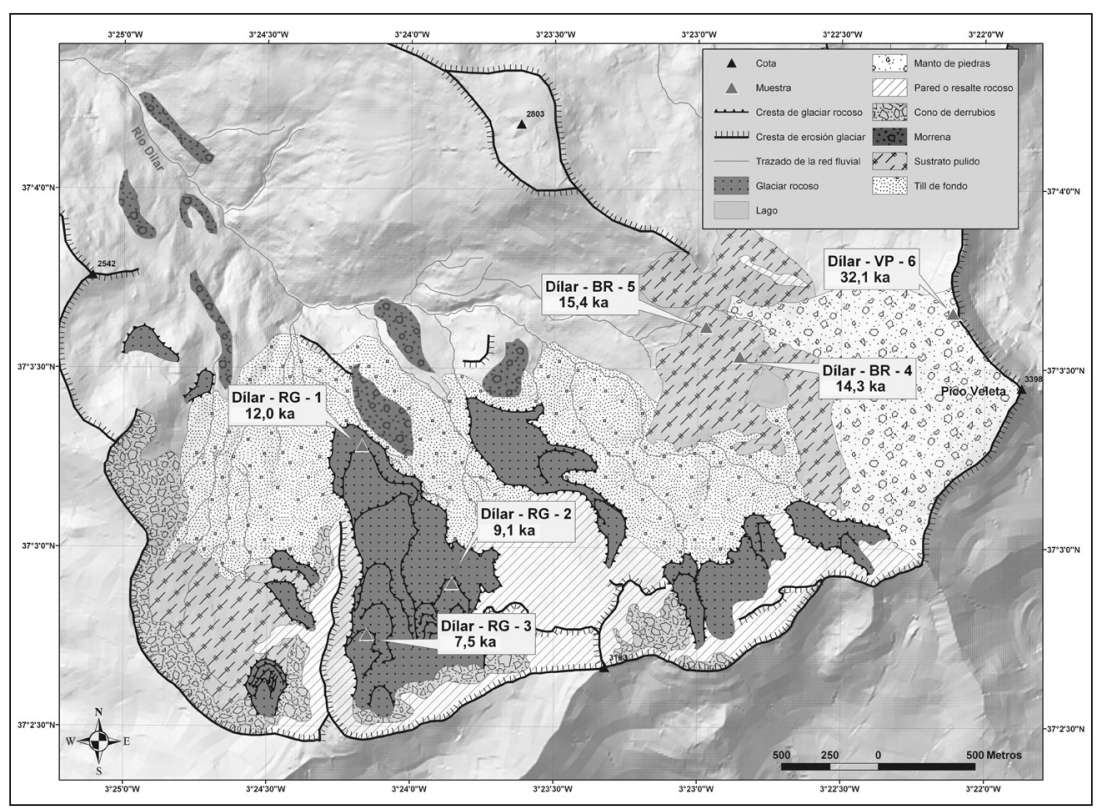

\section{Valle de Dílar}

En la cabecera del río Dílar, en la vertiente occidental del Pico del Veleta, también existen morrenas laterales bien conservadas de un gran avance, muy similares a las anteriormente descritas del periodo que hemos denominado UMGL (figura 6). La extensión de estas morrenas laterales indican una longitud del paleoglaciar superior a los $6 \mathrm{~km}$. Igual que en el caso de los valles del sur también hay morrenas de reavances posteriores. Esta lengua glaciar se alimentaba, fundamentalmente, de los circos situados en la vertiente septentrional del valle de Dílar, donde se conservan umbrales rocosos con pulimiento glaciar y espectaculares formaciones de glaciares rocosos, algunos de ellos superpuestos en varias generaciones (Cascajar del Cartujo) superando los $2 \mathrm{~km}$ de longitud. También aquí, todos los glaciares rocosos son relictos y están poco alterados por la erosión posterior.

La morfología de los glaciares rocosos del Cascajar del Cartujo es diversa en relación con su distancia a la cabecera. En sus partes distales, predomina una morfología caótica, característica de colapsos o termokarst, con numerosas depresiones, mientras que en las cabeceras y lenguas más jóvenes la morfología es mucho más regular, con crestas y surcos paralelos, sin áreas de subsidencia.

La alimentación del glaciar de Dílar desde el este del valle fue mínima. De hecho, en el tramo superior de la ladera del Pico del Veleta no quedan restos de formas glaciares. Por el contrario, el substrato aparece resquebrajado y recubierto por grandes campos de bloques (Lastrones del Veleta), lo que permite plantear la hipótesis de un posible comportamiento temporal del referido pico del Veleta como «hörn». 


\section{Valle de Monachil}

La cabecera del río Monachil, al noroeste del Pico del Veleta, conserva también claras muestras de erosión glaciar, aunque la mayor parte del área ha sido alterada por las instalaciones de la estación de esquí (figuras 7 y 8). Únicamente se conservan restos de formaciones

Figura 7

\section{MORRENAS DEL UMGL EN EL VALLE DE MONACHIL Y MUESTRAS MONA-M-1 Y 2}

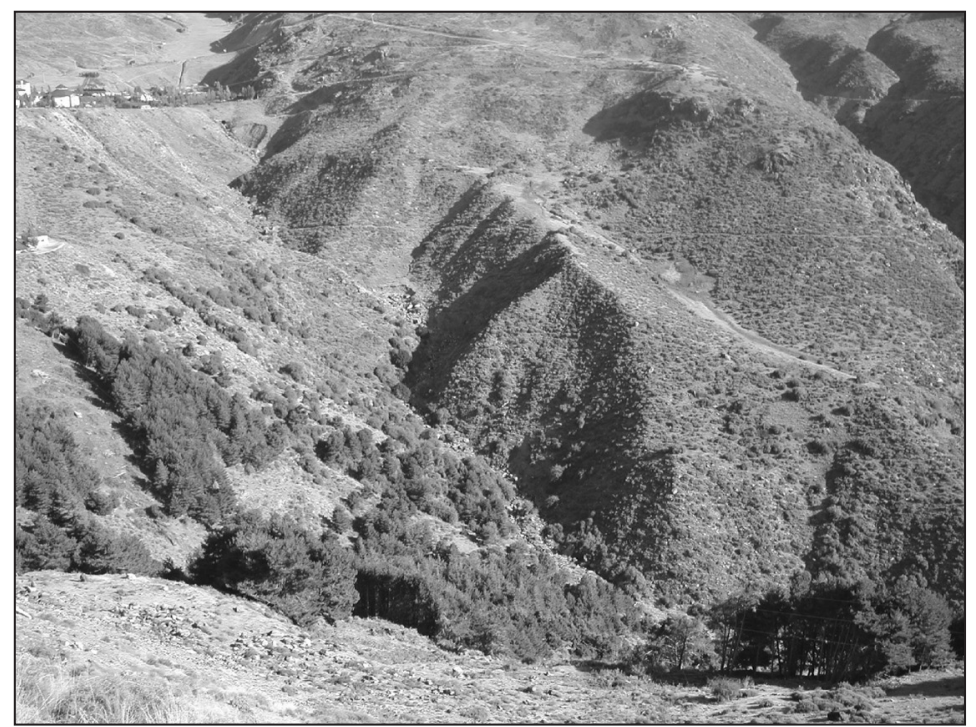

Figura 8

ESQUEMA GEOMORFOLÓGICO DEL VALLE DE MONACHIL, MUESTRAS Y RESULTADOS

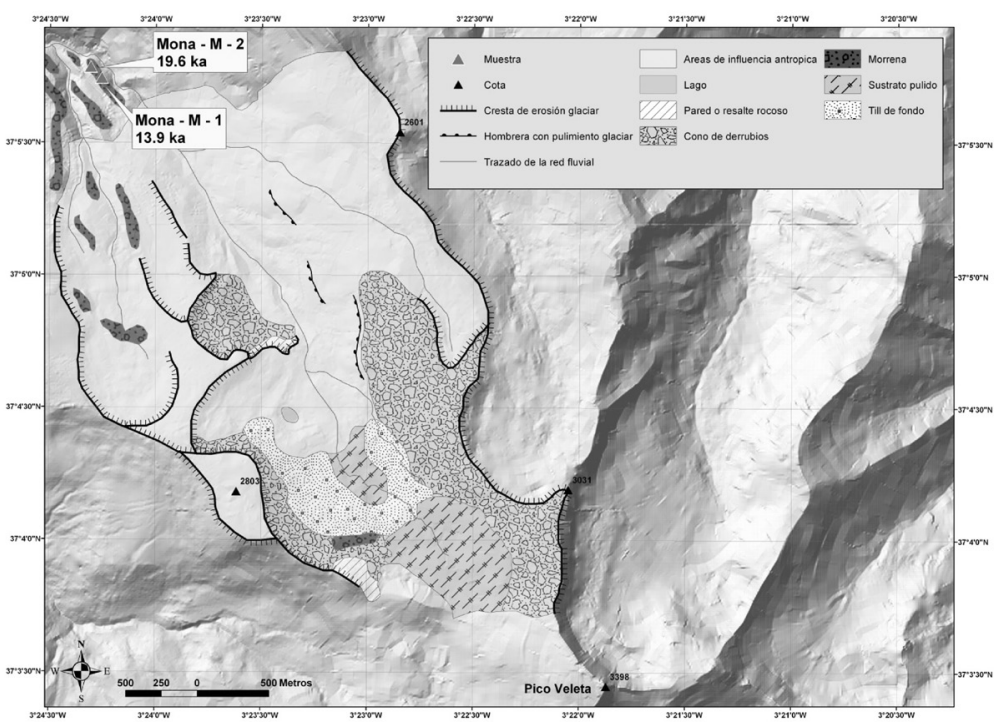


morrénicas en buen estado fuera de los límites de esta estación, justo aguas abajo del núcleo urbano de Pradollano. Se trata de morrenas de pequeña entidad, similares a las fases de reavance posterior al UMGL en los otros valles analizados. En este caso, las morrenas del UMGL están deterioradas por la erosión fluvial y antrópica.

\section{RESULTADOS}

Con los criterios establecidos en los dos apartados anteriores se seleccionaron las localidades donde se obtuvieron las muestras de los cuatro barrancos objeto de estudio: Dílar y Monachil y Mulhacén y Río Seco, estos dos últimos en el valle de Poqueira. De Dílar (DILAR) se muestreo la cabecera, mientras que de Mulhacén (MULH), Río Seco (SECO) y Monachil (MONA) cabecera y valle. Las muestras, en número de 19, se obtuvieron de bloques de morrenas (M), sustrato rocoso afectado por la abrasión glaciar (BR), bloques de glaciares rocosos $(\mathrm{RG})$ y en un sólo caso se recogió muestra del sustrato rocoso, dentro de un área que no tiene huellas de abrasión glaciar, cerca de la cumbre del Pico del Veleta (VP).

\section{Barranco de Mulhacén}

En el barranco de Mulhacén se obtuvieron dos muestras de la formación morrénica que hemos denominado UMGL, una de cada morrena lateral (MULH-M-1 y MULH-M-2), situadas a unos $2.500 \mathrm{~m}$ de altitud. Se muestrearon también dos escalones rocosos consecutivos fijados en torno a los $3000 \mathrm{~m}$ de altitud, que conservaban intensos pulimientos glaciares (MULH-BR-3 y MULH-BR-4). Por último, se recogieron dos muestras de una morrena de nevero situada en la base de la pared de cabecera del circo (MULH-M-5 y MULH-M-6).

Los resultados obtenidos del barranco de Mulhacén (tabla 1 y figura 9) nos llevan a rechazar la datación de la muestra MULH-M-1 (11,6 ka) por carecer de toda lógica geomorfológica, al ser posterior a los resultados de muestras procedentes de formas del relieve glaciar situadas mucho más próximas a la cabecera del valle (como sucede en los casos MULH-BR-3 y MULH-BR-4). Evidentemente, este bloque morrénico ha debido ser erosionado con intensidad o removido posteriormente a su deposición glaciar. Distinto es el resultado de la muestra MULH-M-2, ubicada en la misma situación geomorfológica que la anterior, y que da una fecha de $30 \mathrm{ka}$. Aunque el dato es muy indicativo, al tratarse sólo

Tabla 1

RESULTADOS DE LAS MUESTRAS ${ }^{36}$ CL DEL VALLE DE MULHACÉN

\begin{tabular}{|c|c|c|c|c|c|}
\hline MUESTRA & $\begin{array}{c}\text { EDAD } \\
\text { ka }\end{array}$ & LATITUD & LONGITUD & $\begin{array}{c}\text { ALTITUD } \\
\text { m s.n.m. }\end{array}$ & UNIDAD GEOMOFOLÓGICA \\
\hline MULH-M-1 & 11,6 & $37^{\circ} 01^{\prime} 48,10^{\prime \prime} \mathrm{N}$ & $3^{\circ} 19^{\prime} 46,90^{\prime \prime} \mathrm{W}$ & 2.461 & Bloque morrénico \\
\hline MULH-M-2 & 30,0 & $37^{\circ} 01^{\prime} 40,10^{\prime \prime} \mathrm{N}$ & $3^{\circ} 19^{\prime} 37,60^{\prime \prime} \mathrm{W}$ & 2.448 & Bloque morrénico \\
\hline MULH-BR-3 & 12,0 & $37^{\circ} 02^{\prime} 50,05^{\prime \prime} \mathrm{N}$ & $3^{\circ} 19^{\prime} 26,47^{\prime \prime} \mathrm{W}$ & 2.912 & Umbral rocoso glaciar \\
\hline MULH-BR-4 & 14,6 & $37^{\circ} 03^{\prime} 01,71^{\prime \prime} \mathrm{N}$ & $3^{\circ} 19^{\prime} 27,49^{\prime \prime} \mathrm{W}$ & 3.004 & Umbral rocoso glaciar \\
\hline MULH-M-5 & 14,7 & $37^{\circ} 03^{\prime} 10,90^{\prime \prime} \mathrm{N}$ & $3^{\circ} 19^{\prime} 47,88^{\prime \prime} \mathrm{W}$ & 3.036 & Bloque morrena de nevero \\
\hline MULH-M-6 & 8,7 & $37^{\circ} 03^{\prime} 10,27^{\prime \prime} \mathrm{N}$ & $3^{\circ} 19^{\prime} 50,48^{\prime \prime} \mathrm{W}$ & 3.090 & Bloque morrena de nevero \\
\hline
\end{tabular}


de una muestra no resulta concluyente. Los resultados obtenidos en los umbrales rocosos (MULH-BR-3 y MULH-BR-4) son próximos $(12,0-14,6)$ y altamente fiables debido al buen estado de conservación que muestra el pulimiento glaciar donde se obtuvieron.

También dentro de la lógica geomorfológica se encuentran los resultados del depósito de la morrena de nevero que cierra la laguna de la Caldera (MULH-M-5 y MULH-M-6), en la base del circo y cerca de sus paredes. Una de las muestras refleja 14,7 ka y la otra 8,7 ka. La primera, viene a ser contenporánea a la abrasión de los umbrales rocosos donde se asienta, lo que viene a significar que esta morrena pudo empezarse a formar apenas el hielo se retiró del umbral y se refugió en el circo. La segunda muestra, más reciente y fijada $8 \mathrm{~m}$ por encima de la anterior, podría asociarse a algún episodio de caida de bloques de la pared limítrofe del circo durante tiempos más cercanos a nosotros, cuando aún los hielos y las nieves ocupaban parte de la cabecera del barranco.

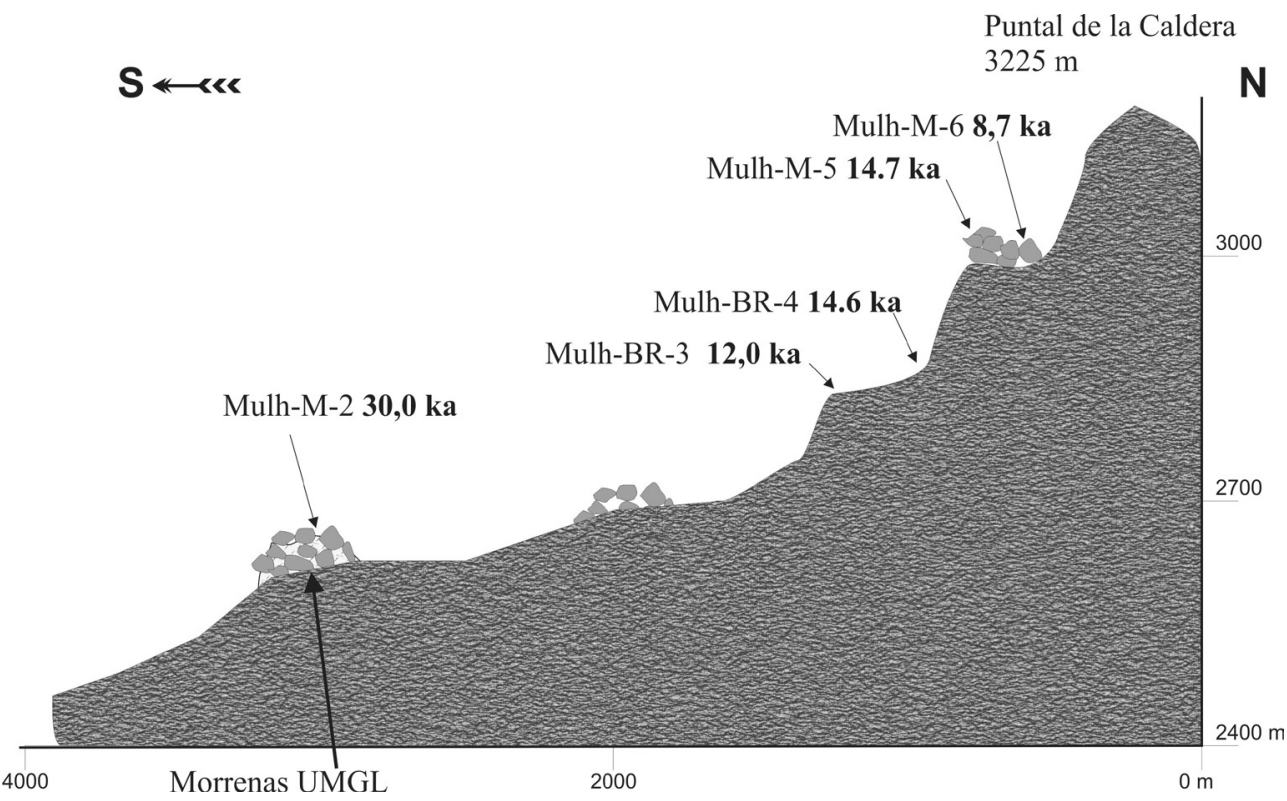

\section{Barranco de Río Seco}

En el barranco de Río Seco se tomaron también muestras de las morrenas del estadio correspondiente al UMGL, (SECO-M-1, SECO-M-2 y SECO-M-3), a una altitud de 2.450 $\mathrm{m}$, aproximadamente. Igualmente, de un umbral rocoso afectado por un intenso y bien conservado pulimiento glaciar (SECO-BR-4) y, por último, de un bloque del glaciar rocoso principal, que se apoya en dicho umbral (SECO-GR-5) (tabla 2 y figura 10). Estas últimas muestras se instalan a una altitud próxima a los $3.000 \mathrm{~m}$. 
Tabla 2

RESULTADOS DE LAS MUESTRAS ${ }^{36} \mathrm{CL}$ DEL VALLE DEL RÍO SECO

\begin{tabular}{|c|c|c|c|c|c|}
\hline MUESTRA & $\begin{array}{c}\text { EDAD } \\
\text { ka }\end{array}$ & LATITUD & LONGITUD & $\begin{array}{c}\text { ALTITUD } \\
\text { m s.n.m. }\end{array}$ & UNIDAD GEOMORFOLÓGICA \\
\hline SEC0-M-1 & 14,2 & $37^{\circ} 01^{\prime} 43,40^{\prime \prime} \mathrm{N}$ & $3^{\circ} 20^{\prime} 08,50^{\prime \prime} \mathrm{W}$ & 2.423 & Bloque morrénico \\
\hline SECO-M-2 & 13,4 & $37^{\circ} 01^{\prime} 42,40^{\prime \prime} \mathrm{N}$ & $3^{\circ} 20^{\prime} 11,10^{\prime \prime} \mathrm{W}$ & 2.437 & Bloque morrénico \\
\hline SECO-M-3 & 19,0 & $37^{\circ} 01^{\prime} 40,40^{\prime \prime} \mathrm{N}$ & $3^{\circ} 20^{\prime} 12,40 \prime \prime \mathrm{W}$ & 2.446 & Bloque morrénico \\
\hline SECO-BR-4 & 12,7 & $37^{\circ} 02^{\prime} 58,03 ” \mathrm{~N}$ & $3^{\circ} 20^{\prime} 41,63^{\prime \prime} \mathrm{W}$ & 2.984 & Umbral rocoso glaciar \\
\hline SEC0-RG-5 & 9,6 & $37^{\circ} 02^{\prime} 46,75^{\prime \prime} \mathrm{N}$ & $3^{\circ} 20^{\prime} 37,84^{\prime \prime} \mathrm{W}$ & 2.895 & Bloque glacial rocoso \\
\hline
\end{tabular}

Al igual que ocurrió en el barranco de Mulhacén, las muestras de bloques morrénicos relacionadas con el UMGL ofrecen resultados alternativos. Dos de los bloques se sitúan en torno a los 13-14 ka, lo que no sería coherente debido a la fijación altitudinal de sus morrenas. La otra (SECO-M-3), vuelve a tener más lógica, con 19 ka, pero, al ser sólo una, es orientativa. De nuevo las muestras de mayor altitud mantienen la lógica geomorfológica y sus resultados son muy similares a las del barranco de Mulhacén. El umbral rocoso (SECOBR-4) tiene una edad próxima al del barranco de Mulhacen, con 12,7. El glaciar rocoso (SECO-RG-4) dejó de ser activo hace 9,6 ka, pero no sabemos cuando se formó, ya que su movimiento, aunque fuera lento, impediría la permanente exposición de sus bloques a la radiación. En cualquier caso, sería con posterioridad a la deglaciación del umbral en el que se apoya $(12,7 \mathrm{ka})$.

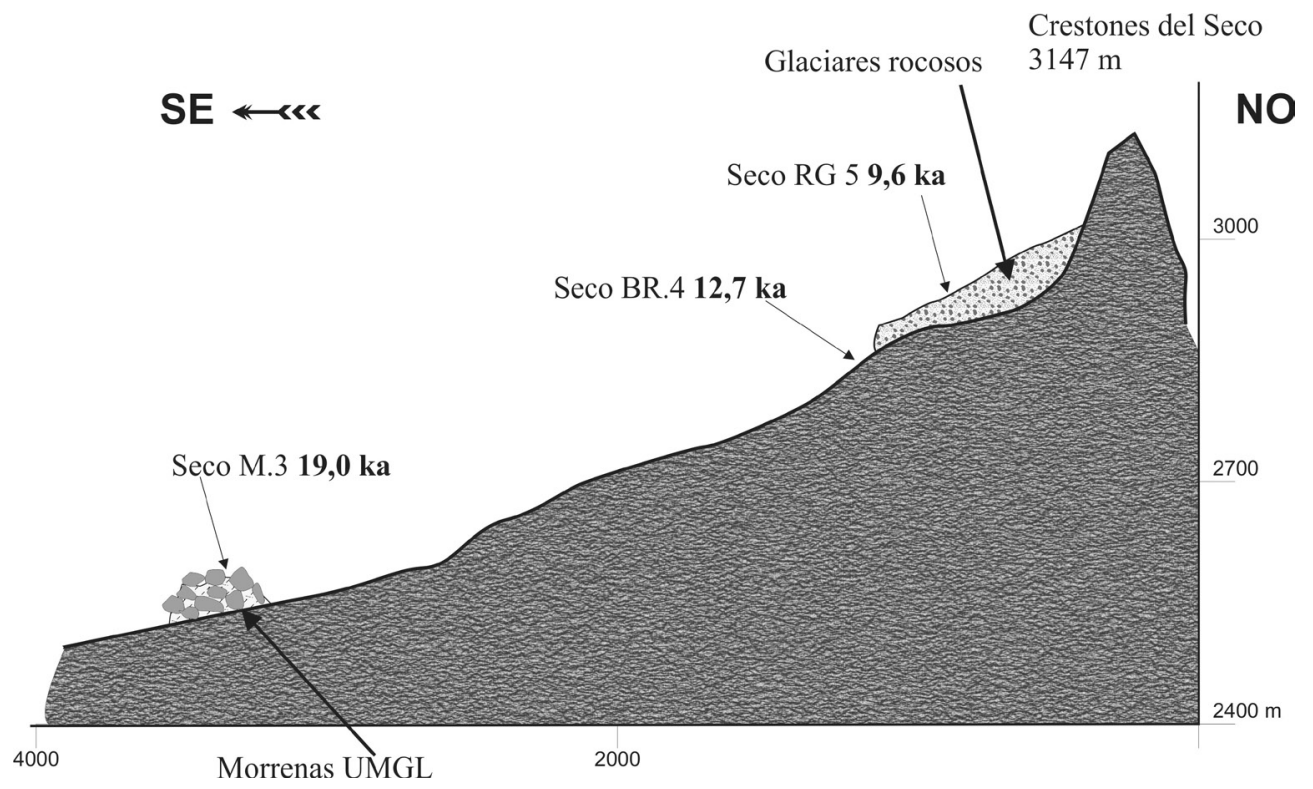




\section{Valle de Dilar}

En la vertiente meridional de la cabecera del río Dílar, en el llamado Cascajar del Cartujo, se obtuvieron tres muestras de bloques de tres lenguas de glaciares rocosos escalonados en altura (tabla 3 y figura 11). La primera, cerca del frente de la lengua más antigua y larga, a 1.500 metros de la cumbre (DILAR-RG-1); la segunda, en el frente de una lengua intermedia, a unos $550 \mathrm{~m}$ de la cumbre (DILAR-RG-2). Y la más alta, en el frente de la lengua más reciente, a sólo $400 \mathrm{~m}$ de la cumbre (DILAR-RG-3). Estos glaciares rocosos descansan sobre rellanos del substrato que, más hacia el este, se prolongan y se muestran pulidos por la acción glaciar. De ellos se recogieron dos muestras (DILAR-BR-4 y DILAR-BR-5). Por último, ya por encima del dominio glaciado, en las proximidades del Pico del Veleta, en el sector que podría haber funcionado como un «hörn», también se obtuvo una muestra (DILAR-VP-6).

Tabla 3

RESULTADOS DE LAS MUESTRAS 36CL DEL VALLE DE DÍLAR

\begin{tabular}{|c|c|c|c|c|c|}
\hline MUESTRA & EDAD ka & LATITUD & LONGITUD & $\begin{array}{c}\text { ALTITUD } \\
\text { m s.n.m. }\end{array}$ & UNIDAD GEOMORFOLÓGICA \\
\hline DILAR-RG-1 & $\mathbf{1 2 , 0}$ & $37^{\circ} 03^{\prime} 12,57^{\prime \prime} \mathrm{N}$ & $3^{\circ} 24^{\prime} 14,25^{\prime \prime} \mathrm{W}$ & 2.583 & Bloque glacial rocoso \\
\hline DILAR-RG-2 & $\mathbf{9 , 1}$ & $37^{\circ} 02^{\prime} 49,57^{\prime \prime} \mathrm{N}$ & $3^{\circ} 23^{\prime} 55,41^{\prime \prime} \mathrm{W}$ & 2.784 & Bloque glacial rocoso \\
\hline DILAR-RG-3 & $\mathbf{7 , 5}$ & $37^{\circ} 02^{\prime} 40,99^{\prime \prime} \mathrm{N}$ & $3^{\circ} 24^{\prime} 13,17^{\prime \prime} \mathrm{W}$ & 2.870 & Bloque glacial rocoso \\
\hline DILAR-BR-4 & $\mathbf{1 4 , 3 0}$ & $37^{\circ} 03^{\prime} 32,66^{\prime} \mathrm{N}$ & $3^{\circ} 23^{\prime} 02,38^{\prime \prime} \mathrm{W}$ & 2.828 & Umbral rocoso glaciar \\
\hline DILAR-BR-5 & $\mathbf{1 5 , 4 0}$ & $37^{\circ} 03^{\prime} 27,70^{\prime} \mathrm{N}$ & $3^{\circ} 22^{\prime} 55,37^{\prime} \mathrm{W}$ & 2.873 & Umbral rocoso glaciar \\
\hline DILAR-VP-6 & $\mathbf{3 2 , 1 0}$ & $37^{\circ} 03^{\prime} 34,99^{\prime} \mathrm{N}$ & $3^{\circ} 22^{\prime} 10,77^{\prime \prime} \mathrm{W}$ & 3.211 & Umbral no glaciado \\
\hline
\end{tabular}

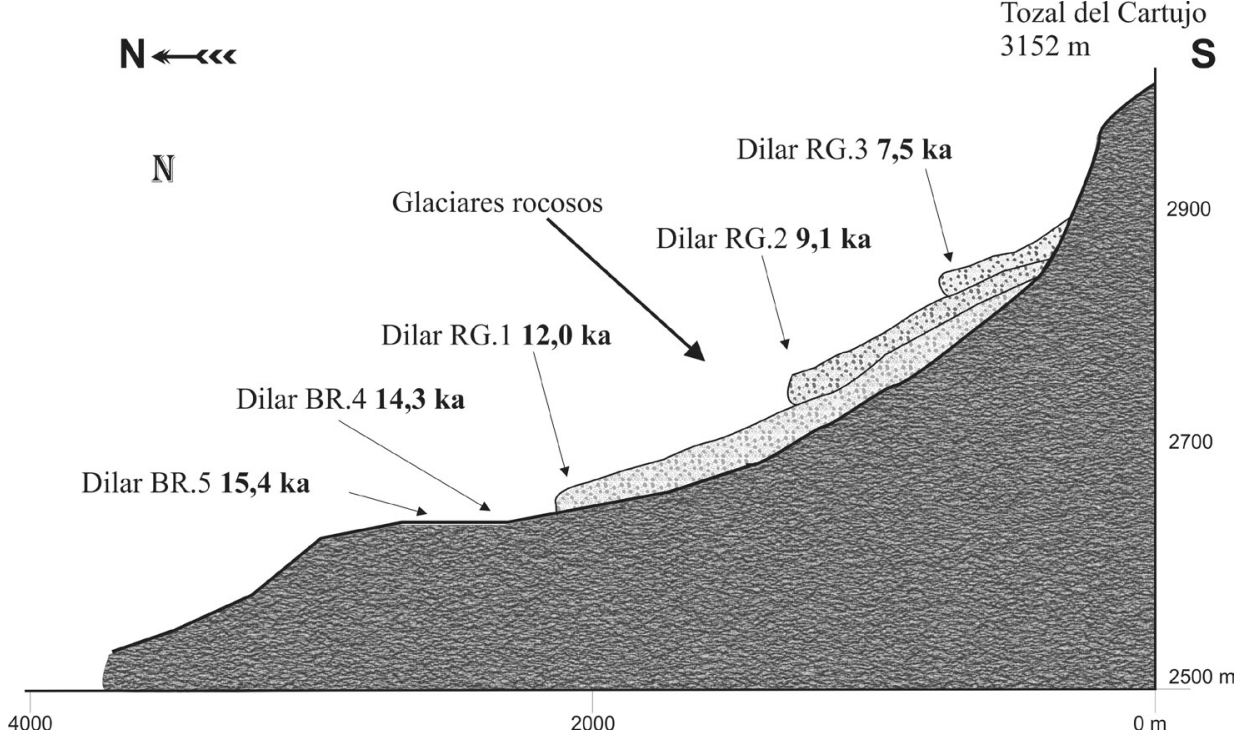


Los resultados de todas estas muestran resultan coherentes. Los umbrales rocosos (DILAR-BR-4 y DILAR-BR-5) dan fechas muy similares entre si, 14,3 y 15,4 ka, respectivamente, muy en consonancia con los umbrales de Río Seco y Mulhacén. Los glaciares rocosos presentan fechas más recientes, como es lógico, y bien secuenciadas entre ellas. El más antiguo (DILAR-RG-1) pudo tener un máximo de duración entre 1 y 2 ka, pues quedó inactivo en 12,0 ka. Las otras dos lenguas también se suceden con intervalos de $2 \mathrm{ka}$. La más reciente (DILAR-RG-3) se estabilizó hace 7,5 ka. La muestra recogida cerca del Pico del Veleta (DILAR-VP-6) podría confirmar que este pináculo actuaría como hörn, al menos durante la última glaciación, pues ofrece una edad de 32,1 ka.

\section{Valle de Monachil}

Por último, para completar la serie del conjunto de valles analizados, se seleccionaron dos muestras de bloques pertenecientes a sistemas morrénicos del que hemos denominado UMGL en el valle del Monachil (MONA-M-1 y MONA-M-2), aguas abajo del área alterada por la estación de esqui (tabla 4). Los resultados de las dataciones obtenidas ofrecen dudas, sobre todo en la muestra (MONA-M-1) que formando parte del mismo segmento de la morrena que la muestra (MONA-M-2) y distante entre ellas solo unos $150 \mathrm{~m}$, resulta demasiado reciente si la comparamos con las fechas obtenidas en otras muestras instaladas en morrenas de mayor altitud (SECO-M-3). En cuanto a la MONA-M-2, su edad (19,6 ka) pudiera parecer más correcta.

Tabla 4

RESULTADOS DE LAS MUESTRAS ${ }^{36}$ CL DEL VALLE DE MONACHIL

\begin{tabular}{|c|c|c|c|c|c|}
\hline MUESTRA & $\begin{array}{c}\text { EDAD } \\
\text { ka }\end{array}$ & LATITUD & LONGITUD & $\begin{array}{c}\text { ALTITUD } \\
\text { m s.n.m. }\end{array}$ & UNIDAD GEOMORFOLÓGICA \\
\hline MONA-M-1 & $\mathbf{1 3 , 9 0}$ & $37^{\circ} 05^{\prime} 40,17^{\prime \prime} \mathrm{N}$ & $3^{\circ} 24^{\prime} 19,49^{\prime} \mathrm{W}$ & 2.006 & Bloque morrénico \\
\hline MONA-M-2 & $\mathbf{1 9 , 6 0}$ & $37^{\circ} 05^{\prime} 42,70^{\prime} \mathrm{N}$ & $3^{\circ} 24^{\prime} 22,64^{\prime} \mathrm{W}$ & 1.975 & Bloque morrénico \\
\hline
\end{tabular}

\section{DISCUSIÓN}

Como en la mayoría de las montañas europeas glaciadas también en Sierra Nevada quedan muestras en su relieve de una reciente gran expansión glaciar. Se trata de morrenas bien delimitadas, de gran tamaño y bien conservadas, formadas en un periodo que en este trabajo hemos denominado UMGL por su similitud con las formas morrénicas de otras montañas europeas que se han considerado contemporáneas con el Último Máximo Glaciar Global (UMGG). Sin embargo, el estudio geomorfológico realizado recientemente en Sierra Nevada demuestra que existen restos de formaciones morrénicas más antiguas al UMGL en diversos valles. Por su grado de alteración estas formaciones no son válidas para su datación por elementos cosmogénicos.

Los datos obtenidos en este trabajo tampoco proporcionan información concluyente respecto a la edad del UMGL. En cada valle muestreado algunos bloques seleccionados de las morrenas instaladas en las vertientes indican fechas recientes (SECO-M-2; MONA-M-1), 
poco compatibles con los resultados muy fiables de los umbrales rocosos situados cerca de las cabeceras de los antiguos glaciares (SECO-BR-4; DILAR-BR-5). Sin embargo, otros bloques de morrenas, próximos a los anteriores, denotan edades más antiguas (MONAM-2; SECO-M-3). Este hecho no sorprende, si tenemos en cuenta la actividad morfogénica de laderas que impera en las cumbres de Sierra Nevada y la naturaleza del material que conforma las morrenas, sensible a su desestabilización. Ello nos lleva a pensar en posibles movimientos de determinados bloques mal anclados en las morrenas, lo que pudiera haber conducido a falsear sus edades.

Las edades de las muestras MONA-M-2 (19,6 ka), SECO-M-3 (19,0 ka) y MUL-M-2 $(30,0 \mathrm{ka})$, de tres segmentos de morrenas de valles diferentes, nos parecen muy fiables y de no haber existido movimientos de los bloques donde se obtuvieron sus edades podrían inducir a considerar que el UMGL de Sierra Nevada estaría próximo al UMGG. Sería, probablemente, similar a otras montañas ibéricas y del sur de Europa donde se han obtenido resultados muy parecidos también por métodos cosmogénicos, como se ha apuntado en la introducción de este trabajo. Al igual que en estas montañas, el máximo avance local en Sierra Nevada sería anterior al UMGG, pues en el 19 ka, probablemente, se operaría el comienzo del retroceso y reclusión de las lenguas glaciares hacia sus circos. Esta hipótesis de un ultimo máximo avance local anterior al LGM concuerda con otros proxys paleoclimáticos que señalan para este periodo pre-LGM un clima frío y húmedo para la península Ibérica (López-García, 2010; Moreno et al., 2010a, 2010b), mientras que para los mismos años del UMGG parece persistir aún un clima todavía muy frío, pero árido con niveles mínimos en los lagos (Morellón et al., 2009; Vegas et al., 2010).

En Sierra Nevada, entre las morrenas del UMGL y los umbrales rocosos más altos, existen diferentes generaciones de segmentos de morrenas de reavance, muy dispersas y no bien correlacionables entre los distintos valles. De ellas aún no disponemos de datos precisos de su cronología, pero sí resultan concluyentes los obtenidos en umbrales rocosos con pulimiento glaciar. En todos los valles y en todos los casos dan resultados muy próximos (MULH-BR-3; DILAR-BR-4), por lo que se puede afirmar, sin lugar a duda, que la gran deglaciación de Sierra Nevada estaría concluida en torno a los 14 ka, quedando los hielos en ese momento encerrados en los sectores más favorables de los circos. Esta fecha coincide con el máximo retroceso glaciar a nivel planetario (Clark et al., 2009), con los datos obtenidos en los Pirineos (González-Sampériz et al., 2006; Pallàs et al., 2006, 2010; Delmas et al., 2008; Sancho et al., 2008), en el noroeste de la Península Ibérica (Fernández-Mosquera et al., 2000; Cowton et al., 2009) y centro de ella (Palacios et al., 2011), así como con otras montañas del sur de Europa, como en los Alpes (Ivy-Ochs et al., 2004, 2006, 2008; Preusser, 2004; Kerschner y Ivy-Ochs, 2008) y oeste del Mediterráneo (Akçar et al., 2007, 2008; Monegato et al., 2007; Sarikaya et al., 2008; Zahno et al., 2010).

Deglaciados los valles de Sierra Nevada aún permanecieron pequeños focos glaciares o grandes neveros en el interior de los circos más altos, como el que ocupaba la actual laguna de la Caldera, en la cabecera del barranco de Mulhacén. Sin embargo, lo más relevante en tales circos, sobre todo en aquéllos de orientacion norte o noreste y este sureste, fue el desarrollo de distintos sistemas de glaciares rocosos. Su formación fue prácticamente contemporanea al proceso de deglaciación, pues el más antiguo y de mayor extensión de los analizados (DILAR-RG-1) quedó inactivo hace 12 ka, 2 ka después de la desaparición del glaciar de 
Dílar. En su conjunto, estos glaciares rocosos de Dílar formaban cuerpos lobulados a partir de la sucesión de arcos escalonados dispuestos según la pendiente. La estabilización de los frentes de estos arcos se fija entre los 12,0 ka para el más bajo $(2.635 \mathrm{~m})$ y los 7,5 ka para el más elevado $(2.837 \mathrm{~m})$. El cese de actividad de las lenguas más antiguas y la superposición de otras nuevas sobre ellas, no se debe considerar fruto de diferentes ciclos climáticos.

\section{Sobre el origen de los glaciares rocosos y sus condiciones de formación}

Como ya se señaló, en la actualidad sólo existe en Sierra Nevada un glaciar rocoso activo, a unos $3.100 \mathrm{~m}$ de altitud, es decir, por debajo de la isoterma de $\operatorname{los} 0^{\circ} \mathrm{C}$. Su origen deriva de la reciente desaparición del glaciar que existió al pie de la pared norte del pico del Veleta durante la Pequeña Edad de Hielo (Gomez-Ortiz et al., 2008). El glaciar, en la medida que iba retrocediendo, quedó sepultado por los bloques caídos y desprendidos procedentes de sus paredes rocosas limítrofes y en el tiempo fueron adoptando, paulatinamente, formas de flujo. El manteniendo actual del glaciar rocoso se debe a la prolongada persistencia de la nieve en el suelo, que mitiga el efecto de la radiación externa en su interior (Gomez-Ortiz et al., 2001 y Tanarro et al., 2001). En las últimas décadas, este cometido protector del manto nival en verano resulta determinante ( Gómez Ortiz et al., 2008; Salvador-Franch et al., 2010, 2011; Tanarro et al., 2010).

Si comparamos la existencia del glaciar rocoso actual y los que se desarrollaron tras la gran deglaciación de la Sierra, se encuentran coincidencias en muchos aspectos: instalación en el fondo de los circos glaciares, localización bajo paredes muy activas, orientación propicia a baja insolación pero favorable a la sobrealimentación nival, etc. Según todo ello, los glaciares rocosos de Sierra Nevada no parece que obedecieran fundamentalmente a condiciones climáticas periglaciares muy frías y secas con generalización de permafrost, sino que serían fruto, sobre todo, de unas condiciones climáticas finiglaciares muy favorables a procesos intensos de gelifracción, al aporte de nieves y a la existencia de aridez. En estas condiciones, el hielo glaciar en retroceso de los circos de Sierra Nevada, instalado al amparo de paredes, tendería a quedar atrapado bajo espesos mantos de bloques y evolucionaría, primero, como glaciar negro y luego, en bloque todo el cuerpo, como glaciar rocoso, pudiéndose clasificar éste como glacier-derived rock glaciers (Humlum, 1999, 2000). De esta forma, el factor geomorfológico (actividad de la pared-pendiente) sería determinante en el suministro de material clástico, como también el aporte de nieve, que contribuiría a dar plasticidad al cuerpo helado y al manto detrítico, además de amortiguar la radiación externa (Jonhson, 1987; Fort, 2003; Johnson, et al., 2007).

Muchos glaciares rocosos estudiados en épocas presentes en áreas frías y áridas actuales han servido para usarlos como indicativo de la existencia de permafrost (Frauenfelder y Kääb, 2000; French, 2007). Para que se dieran este tipo de glaciares rocosos sería necesario que la temperatura media anual de esas áreas (MAAT) debería ser igual o inferior a $-2^{\circ} \mathrm{C}$ (Barsch, 1996; Haeberli, 1985). Pero como se ha indicado anteriormente, en la actualidad esa temperatura no se alcanza ni en las cumbres más altas de la Sierra, a $3.400 \mathrm{~m}$, donde no baja de $\operatorname{los}-0,4^{\circ} \mathrm{C}$ (Salvador-Franch et al., 2010, 2011) y sin embargo, existe un glaciar rocoso activo en ese ambiente térmico, si bien su origen está claramente derivado de la degradación de un glaciar previo. 
Los glaciares rocosos que se convirtieron en relictos a principios del Holoceno en Sierra Nevada tienen una altitud mínima de $2.500 \mathrm{~m}$. La MAAT actual para esa altitud es de $4^{\circ} \mathrm{C}$ aproximadamente (Montávez et al., 1996). Es decir, que si esos glaciares rocosos se correspondieran a una situación puramente periglaciar debería haber existido en el momento de su formación una MAAT inferior en $6^{\circ} \mathrm{C}$ a la actual. Los proxys paleoclimáticos derivados de estudios próximos a Sierra Nevada no reflejan esas condiciones térmicas, en absoluto. Por ejemplo, en las cuevas de Padul y Carihüela, situadas en las inmediaciones de Sierra Nevada, análisis polínicos indican que el periodo de la existencia de los glaciares rocosos en las cumbres, coincide con la expansión del bosque termófilo en sus laderas, salvo una pequeña crisis fría durante el Dryas Reciente (Fernández et al., 2007). Lo mismo ocurre en el estudio de polen realizado en la perforación en el mar de Alborán, justo al sur de Sierra Nevada, donde salvo la pequeña crisis fría del Dryas Reciente, la existencia de los glaciares rocosos de Sierra Nevada coincide con la gran expansión del bosque termófilo en la costa oeste mediterránea (Combourieu-Nebout et al., 2009; Fletcher et al., 2010). En el centro de la Península Ibérica, en una laguna próxima a Ciudad Real, se ha identificado también a través del polen, un calentamiento generalizado a partir de 16-15 ka, interrumpido por el Dryas Reciente y por tres breves periodos fríos y secos holocenos: 9,2-8,6, 7,5-7 y 5,5-5 ka (Vegas et al., 2010). En cualquier caso, el frío nunca sería tan intenso durante el Dryas Reciente ni las crisis frías posteriores como para propiciar una MAAT inferior en $6^{\circ} \mathrm{C}$ con respecto a la actual. Además, los glaciares rocosos en Sierra Nevada no se forman en relación con esas crisis frías, sino que existen justo después de la deglaciación y algunos ya están relictos antes del Dryas Reciente. Todos estos proxys paleoclimáticos apoyan la hipótesis de que los glaciares rocosos de Sierra Nevada están más en relación con el proceso de la deglaciación y la gran actividad gemorfológica de laderas, que con un clima periglaciar extremo. También la propia morfología superficial de los glaciares rocosos, tipo termokarst en sus áreas más distales, está en consonancia con un origen finiglaciar. Todos estos indicios han hecho rectificar hipótesis iniciales sobre el peso que tuvo una posible crisis climática en el origen de los glaciares rocosos de Sierra Nevada (Palade et al., 2011).

En la actualidad, existen montañas con procesos morfogénicos similares a los descritos en Sierra Nevada en la formación de numerosos glaciares rocosos, como etapa final del proceso de deglaciación. Así se ha indicado, por ejemplo, en el origen de los glaciares rocosos de la Península Tröllaskagi, al norte de Islandia, siempre instalados en fondos de circos glaciares de altas e inestables paredes verticales y como sustitutos de glaciares de la Pequeña Edad de Hielo, ya desaparecidos (Caseldine, 1985; Whalley y Martin, 1994; Whalley et al., 1995; Etzelmüller et al., 2007).

Quizás, uno de los mayores problemas para comprender el significado climático que tuvieron los glaciares rocosos tras la deglaciación de las montañas es la falta de dataciones absolutas. Una de las pocas publicaciones sobre este tema en los Alpes mediterráneos (Cossart et al., 2010) muestra resultados muy similares a los obtenidos en Sierra Nevada: tres generaciones de glaciares rocosos anteriores al Dryas Reciente y falta de conclusiones paleoclimáticas precisas, ya que la localización de los glaciares rocosos analizados está más en dependencia de los fenómenos geomorfológicos que de aquellos otros climáticos, como ya se ha indicado. 


\section{CONCLUSIONES}

La existencia de restos glaciares pertenecientes al Ultimo Máximo Glaciar (LGM) en Sierra Nevada resulta evidente, aunque no es posible saber su edad precisa debido al deficiente estado de conservación que presentan la mayoría de los depósitos morrénicos. La causa de ello radica, sobre todo, en la dinámica erosiva que ha venido afectando a las laderas, que no ha respetado las formas originales. Sin embargo, existen formaciones morrénicas y enclaves rocosos bien conservados equiparables a los que se han identificado como del Ultimo Máximo Glaciar en otras montañas europeas. Sin embargo, a pesar de haber tratado numerosas muestras de ellos, sus resultados cronológicos tampoco son concluyentes. Las muestras que ofrecen mayor seguridad (bloques instalados en morrenas) vienen a mostrar rangos de edades entre 30,0 ka (MULH-M-2, valle de Mulhacén) y 19,0 ka (SECO-M-3, valle de Monachil). Existen, además, en todos los valles de Sierra Nevada formaciones morrénicas posteriores al Último Máximo Glaciar, pero no han sido datadas en este trabajo.

El retroceso de las lenguas glaciares de sus valles hacia cabecera debió resultar rápido, pues las muestras obtenidas en umbrales rocosos, cerca ya de los circos, denotan edades muy similares en todos los valles, lo que valida que este proceso de reclusión de los glaciares, además, fue generalizado. Esto ocurrió a partir de los 15,4 ka (DILAR-BR-5) y los 14,6 (MULH-BR-4), por lo que puede afirmarse que desde entonces la gran masa glaciar de Sierra Nevada debió desaparecer. Sólo en las cabeceras de algunos circos siguieron existiendo pequeños focos glaciares o neveros permanentes, que facilitaron la formaron de importantes sistemas de glaciares rocosos. Las dataciones obtenidas de ellos indican, con seguridad, y por lo que respecta al circo de Dílar, que estuvieron activos entre el 12,0 ka y el 7,5 ka (DILARRG-1; DILAR-RG-3).

Los glaciares rocosos de Sierra Nevada, construidos inmediatamente después de la deglaciación de los valles, se formaron gracias a unas condiciones climáticas muy favorables al desarrollo de procesos de fragmentación mecánica de la roca (crioclastia). Siempre se localizan al pie de paredes escarpadas, bien surtidas en nieves y orientadas al $\mathrm{N} \mathrm{y} \mathrm{NE} \mathrm{y,} \mathrm{en} \mathrm{menor}$ medida al E y SE. La importancia de estos factores disminuye la relevancia de los criterios climáticos respecto a la distribución de la temperatura del suelo. Por este motivo, la existencia de generaciones de glaciares rocosos no resulta ser argumento sólido para delimitar fases frías y secas en esta montaña, como cabría esperar. Los últimos glaciares rocosos en Sierra Nevada pudieron quedar relictos de forma generalizada hace 7,5 ka. En la actualidad, sólo existe uno activo, en el Corral del Veleta, aunque en avanzado proceso regresivo. Su origen se asocia a la desaparición del último foco glaciar de la Sierra, al final de la Pequeña Edad del Hielo.

\section{AGRADECIMIENTOS}

Este trabajo se ha realizado en el marco de actuación de los proyectos de investigación 018/2007 del Organismo Autónomo de Parques Nacionales del Ministerio de Medio Ambiente y el CSO2009-06961 del Ministerio de Ciencia e Innovación. También se integra en el seno de las actividades de los grupos de investigación: Paisatge i paleoambients a la muntanya mediterrània (SGR2009-0898, Generalitat de Catalunya, Universitat de Barce- 
lona) y Geografía Física de Alta Montaña (931562, Universidad Complutense de Madrid). Los autores agradecen la ayuda recibida desde el Parque Nacional de Sierra Nevada.

\section{BIBLIOGRAFÍA}

AKÇAR, N., YAVUZ, V., IVY OCHS, S., KUBIK, P.W., VARDAR, M. y SCHLÜCHTER, C. (2007): «Paleoglacial records from Kavron Valley, NE Turkey: field and cosmogenic exposure dating evidence». Quaternary International, $\mathrm{n}^{\circ}$ 164-165, 170-183.

AKÇAR, N., YAVUZ, V., IVY OCHS, S., KUBIK, P.W., VARDAR, M. y SCHLÜCHTER, C. (2008): «A case for a down wasting mountain glacier during Termination I, Vercenik Valley, NE Turkey». Journal of Quaternary Science, $\mathrm{n}^{\circ}$ 23, 273-285.

BARSCH, D. (1996): Rockglaciers. Heidelberg. Springer Verlag.

BOISSIER, E. (1839): Voyage botanique dans le midi de l'Espagne pendant l'année 1837. Edición facsímil en Fundación Caja de Granada y Universidad de Málaga. Granada, 1995.

CASAS, A. (1943): «Contribución al estudio del glaciarismo cuaternario en Sierra Nevada». Boletín R. Sociedad Española de Historia Natural, V, XLV, 543-567.

CASELDINE, C. (1985): «The extent of some glaciers in Northern Iceland during the Little Ice Age and the nature of recent deglaciation». Geographical Journal, ${ }^{\circ}$ 151, 215-27.

CLARK, P.U., DYKE, A.S., SHAKUN, J.D., CARLSON, A.E., CLARK, J., WOHLFARTH, B., MITROVICA, J.X., HOSTETLER, S.W. y MCCABE, A.M. (2009): «The Last Glacial Maximum». Science, no $325,710-714$.

COMBOURIEU NEBOUT, N. PEYRON, O., DORMOY, I., DESPRAT, S., BEAUDOUIN, C., KOTTHOFF, U. y MARRET F. (2009): «Rapid climatic variability in the west Mediterranean 25,000 years from high resolution pollen data». Climate of the Past, $\mathrm{n}^{\circ}$ 5, 503 521.

COSSART, E., FORT, M., BOURLES, D., CARCAILLET, J., PERRIER, R., SIAME, L. y BRAUCHER, R. (2010): «Climatic significance of glacier retreat and rockglaciers reassessed in the light of cosmogenic dating and weathering rind thickness in Clarée valley (Briançonnais, French Alps)». Catena, $\mathrm{n}^{\circ}$ 80, 204-219.

COWTON, T., HUGHES, P.D. y GIBBARD, P.L. (2009): «Palaeoglaciation of Parque Natural Lago de Sanabria, Northwest Iberia». Geomorphology, nº 108, 282-291.

DELMAS, M., GUNNELL, Y., BRAUCHER, R., CALVET, M. y BOURLÈS, D. (2008): «Exposure age chronology of the last glaciation in the eastern Pyrenees». Quaternary Research, $\mathrm{n}^{\circ}$ 69, 231-241.

ETZELMÜLLER, B., FARBROT, H., GUDMUNDSSON, A., HUMLUM, O., TVEITO, O.E. y BJÖRNSSON, H. (2007): «The Regional Distribution of Mountain Permafrost in Iceland». Permafrost and Periglacial Processes, $\mathrm{n}^{\circ}$ 18, 185-199.

FERNÁNDEZ, S., FUENTES, N., CARRIÓN, J.S., GONZÁLEZ SAMPÉRIZ, P., MONTOYA, E., GIL, G., VEGA TOSCANO, G. y RIQUELME, J.A. (2007): «The Holocene and Upper Pleistocene pollen sequence of Carihuela Cave, southern Spain». Geobios, $\mathrm{n}^{\circ}$ 40, 75-90.

FERNÁNDEZ MOSQUERA, D., MARTI, K., VIDAL ROMANÍ, J.R. y WEIGEL, A. (2000): «Late Pleistocene deglaciation chronology in the NW of the Iberian Peninsula 
using cosmic-ray produced $21 \mathrm{NE}$ in quartz. Nuclear Instruments and Methods». Physics Research $B, \mathrm{n}^{\circ} 172,832-837$.

FLETCHER, W.J., SANCHEZ GOÑI, M.F., PEYRON, O. y DORMOY, I. (2010): «Abrupt climate changes of the last deglaciation. Western Mediterranean forest record». Climate of the Past, $\mathrm{n}^{\circ}$ 6, 245-264.

FORT, M. (2003): «Are high altitude, lava stream-like, debris mixtures all rock glaciers? A perspective from the Western Himalaya». Zeitschrift für Geomorphologie N. F., $\mathrm{n}^{\circ} 130$, 11-29.

FRAUENFELDER, R. y KÄ̈̈B, A. (2000): «Towards a paleoclimatic model of rock glacier formation in the Swiss Alps». Annals of Glaciology, n 31, 281-286.

FRENCH, H.M. (2007): Periglacial Environment. John Wiley \& Sons, 458 pp.

GARCÍA RUIZ, J.M., MORENO, A., GONZÁLEZ SAMPÉRIZ, P., VALERO GARCÉS, B. y MARTÍ BONO, C. (2010): «La cronología del último ciclo glaciar en las montañas del sur de Europa. Una revisión». Cuaternario y Geomorfología, no 24 (1-2), 35-46.

GARCÍA SAINZ, L. (1943): «El glaciarismo cuaternario en Sierra Nevada». Estudios Geográficos, $\mathrm{n}^{\circ} 4,233-254$.

GARCÍA SAINZ, L. (1947): El clima de la España cuaternaria y los factores de su formación. Valencia. Secretariado de Publicaciones de la Universidad de Valencia, 210 pp.

GÓMEZ ORTIZ, A., PALACIOS, D., RAMOS, M., TANARRO, L.M., SCHULTE, L. y SALVADOR-FRANCH, F. (2001): «Location of Permafrost in Marginal Regions: Corral Del Veleta (Sierra Nevada, Spain)». Permafrost and Periglacial Processes, ${ }^{\circ}$ 12, 93-114. GÓMEZ ORTIZ, A., SCHULTE, L., SALVADOR FRANCH, F., SÁNCHEZ, S. y SIMÓN, M. (2002): Geomorphological Map of Sierra Nevada. Glacial and Periglacial Geomorphology. Granada. Consejería de Medio Ambiente de la Junta de Andalucía, Parque Nacional de Sierra Nevada.

GÓMEZ ORTIZ, A., SALVADOR FRANCH, F., SANJOSÉ, J.J., PALACIOS, D., SCHULTE, L. y ATKINSON, A. (2008): «Evolución morfodinámica de un enclave montañoso recién deglaciado: el caso del Corral del Veleta (Sierra Nevada). ¿Consecuencia del Cambio Climático?» Scripta Nova. Revista Electrónica de Geografía y Ciencias Sociales, XII, 270 (26). Disponible en www.ub.edu/geocrit/sn/sn-270/sn-270-26.htm.

GÓMEZ ORTIZ, A., PALACIOS, D., SCHULTE, L., SALVADOR FRANCH, F. y PLANA, J. (2009): «Evidences from historical documents of landscape evolution after Little Ice Age of a mediterranean high mountain area, Sierra Nevada, Spain (Eighteenth to Twentieth centuries)». Geografiska Annaler, nº 91 (4), 279-289.

GONZÁLEZ SAMPÉRIZ, P., VALERO GARCÉS, B., MORENO, A., JALUT, G., GARCÍA RUIZ, J.M., MARTÍ BONO, C., DELGADO HUERTAS, A., NAVAS, A., OTTO, T. y DEDOUBAT, J.J. (2006): «Climate variability in the Spanish Pyrenees during the last 30,000 yr revealed by the El Portalet sequence». Quaternary Research, $\mathrm{n}^{\circ}$ 66, 38-52.

HAEBERLI, W. (1985): «Creep of mountain permafrost. Internal structure and flow of alpine rock glaciers. Mitteilungen der Versuchsanstalt für Wasserbau». Hydrologie und Glaziologie, $\mathrm{n}^{\circ} 77$.

HELLMANN, G. (1881): «Der südlichte Gletscher Europa's». Zeitschrift der Gesellschaft für Erdkunde, $\mathrm{n}^{\mathrm{o}} 16,362-367$. 
HEMPEL, L. (1960): «Límites altitudinales geomorfológicos en Sierra Nevada». Estudios Geográficos, ${ }^{\circ} 78,81-93$.

HUGHES, P.D. y WOODWARD, J.C. (2008): «Timing of Glaciation in The Mediterranean Mountains during The Last Cold Stage». Journal of Quaternary Science, $\mathrm{n}^{\circ} 23$ (6-7), 575-588.

HUGHES, P.D., WOODWARD, J.C. y GIBBARD, P.L. (2006a): «Glacial history of the Mediterranean mountains». Progress in Physical Geography, n 30, 334-364.

HUGHES, P.D., WOODWARD, J.C. y GIBBARD, P.L. (2006b): «Late Pleistocene glaciers and climate in the Mediterranean region». Global and Planetary Change, $\mathrm{n}^{\circ}$ 46, 83-98.

HUGHES, P.D., WOODWARD, J.C. y GIBBARD, P.L. (2007): «Middle Pleistocene cold stage climates in the Mediterranean: New evidence from the glacial record». Earth and Planetary Science Letters, $\mathrm{n}^{\circ} 253,50-56$.

HUMLUM, O. (1999): «The climatic significance of rock glaciers». Permafrost and Periglacial Processes, $\mathrm{n}^{\circ}$ 9, 375-395.

HUMLUM, O. (2000): «The geomorphic significance of rock glaciers: estimates of rock glacier debris volumes and headwall recession rates in West Greenland». Geomorphology, $\mathrm{n}^{\circ} 35,41-67$.

IVY-OCHS, S., SCHÄFER, J., KUBIK, P.W., SYNAL, H. y SCHLÜCHTER, C. (2004): «Timing of deglaciation on the northern Alpine foreland (Switzerland)». Eclogae Geologicae Helvetiae, $\mathrm{n}^{\circ}$ 97, 47-55.

IVY-OCHS, S., KERSCHNER, H., REUTHER, A., MAISCH, M., SAILER, R., SCHAEFER, J., KUBIK, P.W., SYNAL, H. y SCHLÜCHTER, C. (2006): «The timing of glacier advances in the northern European Alps based on surface exposure dating with cosmogenic 10Be, 26Al, 36Cl, and 21Ne» en Situ-Produced Cosmogenic Nuclides and Quantification of Geological Processes (Siame, L.L., Bourlès, D.L., y Brown, E.T., eds.). Geological Society of America Special Paper, ${ }^{\circ}$ 415, 43-60.

IVY OCHS, S., KERSCHNER, H., REUTHER, A., PREUSSER, A., HEINE, K., MAISCH, M., KUBIK, P.W. y SCHLÜCHTER, C. (2008): «Chronology of the last glacial cycle in the European Alps». Journal of the Quaternary Science, $\mathrm{n}^{\circ} 23$ (6-7), 559-573.

JOHNSON, B., THACKRAY, G.D. y VAN KIRK, R. (2007): «The effect of topography, latitude, and lithology on rock glacier distribution in the Lemhi Range, central Idaho, USA» . Geomorphology, ${ }^{\circ}$ 91, 38-50.

JOHNSON, P.G. (1987): «Rock Glacier: glacier debris systems or high-magnitude lowfrequency flows» en Rock Glaciers (Giardino, J., Shroeder, J. y Vitek, J.D., eds.). Londres. Allen and Unwin. 175-192.

KERSCHNER, H. y IVY OCHS, S. (2008): «Palaeoclimate from glaciers: Examples from the Eastern Alps during the Alpine Lateglacial and early Holocene». Global and Planetary Change, $\mathrm{n}^{\circ}$ 60, 58-71.

LAL, D. (1991): «Cosmic-ray labeling of erosion surfaces: in situ production rates and erosion models». Earth and Planetary Science Letters, $\mathrm{n}^{\circ}$ 104, 424-439.

LHENAFF, R. (1977): Recherches géomorphologiques sur les Cordilleres Betiques centrooccidentales (Espagne). Thèse. Université de Lille (France).

LÓPEZ GARCÍA, J.M., BLAIN, H.A., CUENCA BESCOS, G., RUIZ ZAPATA, M.B., DORADO VALIÑO, M., GIL GARCÍA, M.J., VALDEOLMILLOS, A., ORTEGA, A.I., 
CARRETERO, J.M., ARSUAGA, J.A., BERMÚDEZ DE CASTRO, J.M. y CARBONELL, E. (2010): «Palaeoenvironmental and palaeoclimatic reconstruction of the Latest Pleistocene of El Portalón Site, Sierra de Atapuerca, northwestern Spain». Palaeogeography, Palaeoclimatology, Palaeoecology, n 292, 453-464.

MACPHERSON, J. (1875): «De la existencia de fenómenos glaciares en el sur de Andalucía durante la época cuaternaria». Acta de la Sociedad Española de Historia Natural, V, IV, 56-61.

MESSERLI, B. (1965): Beiträge zur Geomorphologie der Sierra Nevada (Andalusien). Zurich. Juris Verlag.

MESSERLI B. (1967): «Die eiszeitliche und die gegenwartige Vertgletscherung im Mittelemeeraum». Geographica Helvetica, n 22, 105-228.

MONEGATO, G., RAVAZZI, C., DONEGANA, M., PINI, R., CALDERONI, G. y WICK, L. (2007): «Evidence of a two-fold glacial advance during the last glacial maximum in the Tagliamento end moraine system (eastern Alps)». Quaternary Research, n 68, 284-302.

MONTÁVEZ, J.P., ROLDÁN, C., RODRÍGUEZ, A. y JIMÉNEZ, J.I. (1996): «Primeros resultados de la climatología de Sierra Nevada» en Conferencia Internacional Sierra Nevada. Granada. Universidad de Granada, 87-99.

MORELLÓN, M., VALERO GARCÉS, B., VEGAS VILARRÚBIA, T., GONZÁLEZ SAMPÉRIZ, P., ROMERO, O., DELGADO HUERTAS, A., MATA, P., MORENO, M., RICO, M. y CORELLA., J.P. (2009): «Late glacial and Holocene palaeohydrology in the western Mediterranean region: the Lake Estanya record (NE Spain)». Quaternary Science Reviews, $\mathrm{n}^{\circ} 28,2582-2599$.

MORENO, A., STOLL, H., JIMÉNEZ SÁNCHEZ, M., CACHO, I., VALERO GARCÉS, B., ITO, E. y EDWARDS, R.L. (2010a): «A speleothem record of glacial (25-11.6 kyr BP) rapid climatic changes from northern Iberian Peninsula». Global and Planetary Change, $\mathrm{n}^{\mathrm{o}} 71,218-231$.

MORENO, A., VALERO GARCÉS, B.., JIMÉNEZ SÁNCHEZ, M., DOMÍNGUEZ, M.J., MATA, P., NAVAS, A., GONZÁLEZ SAMPÉRIZ, P., STOLL, H., FARIAS, P., MORELLÓN, M., CORELLA, P. y RICO, M. (2010b): «The last deglaciation in the Picos de Europa National Park (Cantabrian Mountains, Northern Spain)». Journal of Quaternary Science, $\mathrm{n}^{\circ} 25$ (7), 1076-1091.

OBERMAIER, H. (1916): «Los glaciares cuaternarios de Sierra Nevada». Trabajos del Museo Nacional de Ciencias Naturales (Geología), nº 17, 1-68.

PALACIOS, D., MARCOS, J. y VÁZQUEZ SELEM, L. (2011): «Last Glacial Maximum and Deglaciation of Sierra de Gredos, Central Iberian Peninsula». Quaternary Internacional, $\mathrm{n}^{\circ} 233,16-26$.

PALADE, B., PALACIOS, D. y GÓMEZ ORTIZ., A. (2011): «Los glaciares rocosos de Sierra Nevada y su significado paleoclimático. Una primera aproximación». Cuadernos de Investigación Geográfica, no 37 (2), 95-118.

PALLÀS, R., RODÉS, A., BRAUCHER, R., CARCAILLET, J., ORTUÑO, M., BORDONAU, J., BOURLÉS, D., VILAPLANA, J.M., MASANA, E. y SANTANACH, P. (2006): «Late Pleistocene and Holocene glaciation in the Pyrenees: a critical review and new evidence from 10Be exposure ages, south-central Pyrenees». Quaternary Science Reviews, $\mathrm{n}^{\circ} 25,2937-2963$. 
PALLÀS, R., RODÉS, A., BRAUCHER, R., BOURLÈS, D., DELMAS, M., CALVET, M. y GUNNELL Y. (2010): «Small, isolated glacial catchments as priority targets for cosmogenic surface exposure dating of Pleistocene climate fluctuations, southeastern Pyrenees». Geology, n ${ }^{\circ}$ 38, 891-894.

PASCHINGER, H. (1957). «Las formas glaciares de Sierra Nevada. España». Memorias y Comunicaciones del Instituto Provincial de Barcelona. Barcelona, 81-94.

PHILLIPS, F.M. (2003): «Cosmogenic 36Cl ages of Quaternary basalt flows in the Mojave Desert, California, USA». Geomorphology, nº 53, 199-208.

PHILLIPS, F.M. y PLUMMER, M.A. (1996): «CHLOE: a program for interpreting in-situ cosmogenic nuclide data for surface exposure dating and erosion studies». Radiocarbon, $\mathrm{n}^{\mathrm{o}} 38,98-99$.

PHILLIPS, F.M., STONE, W.D. y FABRYKA-MARTIN, J.T. (2001): «An improved approach to calculating low-energy cosmic-ray neutron fluxes near the land/atmosphere interface». Chemical Geology, n ${ }^{\circ} 175,689-701$.

PONZ, A. (1797): «Relación del viaje que desde Granada hizo á Sierra Nevada D. Antonio Ponz a influxo del Excmo. Sr. Marqués de la Ensenada» en Mensajero económico y erudito de Granada. Granada.

PREUSSER, F. (2004): «Towards a chronology of the Late Pleistocene in the northern Alpine Foreland». Boreas, $n^{\circ} 33,195-210$.

QUELLE, O. (1908): Beiträge zur Kenntnis der spanischen Sierra Nevada. Berlin. Friedrich-Wilheilm Universität.

RUTE, L. de (1889): La Sierra Nevada. Nouvelle Revue Internationale. Paris. Imprimerie Charaire et fils.

SALVADOR FRANCH, F., GÓMEZ ORTIZ, A. y PALACIOS, D. (2010): «Comportamiento térmico del suelo en un enclave de alta montaña mediterránea con permafrost residual: Corral del Veleta (Sierra Nevada, Granada. España)» en Ambientes periglaciares, permafrost y variabilidad climática (Blanco, J.J., de Pablo, M.A. y Ramos, M., Eds.). Alcalá de Henares. Servicio de Publicaciones Universidad de Alcalá de Henares, 61-68.

SALVADOR FRANCH, F., GÓMEZ ORTIZ, A., SALVÀ CATARINEU, M. y PALACIOS, D. (2011): «Caracterización térmica de la capa activa de un glaciar rocoso en medio periglaciar de alta montaña mediterránea. El ejemplo del Corral del Veleta (Sierra Nevada, España)». Cuadernos de Investigación Geográfica, no 37 (2), 25-48.

SÁNCHEZ GÓMEZ, S. (1990): Aplicación del estudio de suelos a la dinámica de la cuenca del río Lanjarón. Relación suelos-geomorfología. Tesis doctoral. Facultad de Ciencias. Granada. Universidad de Granada.

SANCHO, C., PEÑA-MONNÉ, J.L., LEWIS, C., MCDONALD, E. y RHODES, E. (2008): «Actividad glaciar y desarrollo de terrazas durante el Pleiastoceno Superior en PirineosCuenca del Ebro». Geo-Temas, nº 10, 763-766.

SANJOSÉ, J.J., ATKINSON, A., Gómez Ortiz, A. y SALVADOR FRANCH, F. (2007): «Técnicas geodésicas y fotogramétricas aplicadas al análisis de la dinámica y cartografía del glaciar rocoso activo del Corral del Veleta (Sierra Nevada) durante el periodo 20012007». Mapping, $\mathrm{n}^{\mathrm{o}}$ 122, 26-32. 
SARIKAYA, M.A., ZREDA, M., CINER, A. y ZWECK, C. (2008): «Cold and wet Last Glacial Maximum on Mount Sandıras, SW Turkey, inferred from cosmogenic dating and glacier modelling». Quaternary Science Reviews, $\mathrm{n}^{\circ}$ 27, 769-780.

SCHIMPER, P. (1849): Voyage geològique et botànique au Sud de l'Espagne. Paris. Journal de l'Institut.

SERMET, J. (1942): «Sierra Nevada». Estudios Geográficos, nº 9, 727-749.

SORIA, J. y SORIA, M. (1986): «Depósitos de glaciares rocosos en Sierra Nevada». Acta Geológica Hispánica, no 21-22, 123-129.

STONE, J.O.H., EVANS, J.M., FIFIELD, L.K., ALLAN, G.L. y CRESSWELL, R.G. (1998): «Cosmogenic chlorine-36 production in calcite by muons». Geochimica et Cosmochimica Acta, $\mathrm{n}^{\circ}$ 62, 433-454.

TANARRO, L.M., HOELZLE, M., GARCÍA, A., RAMOS, M., GRUBER, S., GÓMEZ ORTIZ, A., PIQUER, M. y PALACIOS, D. (2001): «Permafrost Distribution Modeling in the Mountains of the Mediterranean: Corral del Veleta, Sierra Nevada, Spain». Norsk Geografisk Tidsskrift, n ${ }^{\circ}$ 55, 253-260.

TANARRO, L.M., PALACIOS, D., ZAMORANO, J.J. y GÓMEZ ORTIZ, A. (2010): «Cubierta nival, permafrost y formación de flujos superficiales en un talud detrítico de alta montaña (Corral del Veleta, Sierra Nevada, España)». Cuadernos de Investigación Geográfica, no 36 (2), 39-59.

VEGAS, J., RUIZ ZAPATA, B., ORTIZ, J.E., GALÁN, L., TORRES, T., GIL GARCÍA, M.J., PÉREZ GONZÁLEZ, A. y GALLARDO MILLÁN, J.L. (2010): «Identification of arid phases during the $50 \mathrm{cal}$. ka BP from the Fuentillejo maar-record (Campo de Calatrava Volcanic Field, Spain)». Journal of Quaternary Science, $\mathrm{n}^{\circ} 25$ (7), 1051-1062.

WHALLEY, W.B. y MARTIN E. (1994): «Rock glaciers in Tröllaskagi: their origin and significance» en Environmental Changes in Iceland (Stötter, J. Y Wilhelm, F., eds.). Münchener Geographische Abhandlungen, B, n 12, 289-308.

WHALLEY, W.B., PALMER, C.F., HAMILTON, S.J. y MARTIN, H.E. (1995): «An assessment of rock glacier sliding using 17 years of velocity data - Nautárdalur Rock Glacier, North Iceland». Arctic and Alpine Research, $\mathrm{n}^{\circ} 27,345-351$.

WILLKOMM, M. (1882): Aus den Hochgebirgen von Granada. Viena, 414 pp.

ZAHNO, C., AKÇAR, N., YAVUZ, V., KUBIK, P.W. y SCHLÜCHTER, C. (2010): «Chronology of Late Pleistocene glacier variations at the Uludag Mountain, NW Turkey». Quaternary Science Reviews, $\mathrm{n}^{\circ}$ 29, 1173-1187.

ZREDA, M., ENGLAND, J., PHILliPS, F., ELMORE, D. y SHARMA, P. (1999): «Unblocking of the Nares Strait by Greenland and Ellesmere icesheet retreat 10,000 years ago». Nature, $\mathrm{n}^{\circ} 398,139-142$. 